\title{
154. Realization of Glassy Liquid and Glassy Crystal of Ethanol
}

\author{
By Osamu HaIda, Hiroshi Suga, and Syûzô Seki \\ Department of Chemistry, Faculty of Science, \\ Osaka University, Toyonaka, Osaka
}

(Comm. by Isamu NitTA, M. J. A., Nov. 13, 1972)

Since the heat capacity measurements by G. S. Parks et al. ${ }^{1)}$ and by K. K. Kelley, ${ }^{2)}$ ethanol has been accepted for a long time as one of the typical low molecular weight substance to form glassy state from the supercooled liquid. In this communication, we should like to report that this widely-believed interpretation is not true in its original meaning, and ethanol offers an interesting and valuable example for the deeper understanding of glassy states and glass transition phenomenon: its glassy liquid and glassy crystal states $^{3)}$ could be realized by use of different cooling rates.

Ethanol obtained from Tokyo Kagaku Seiki Co., was purified by dehydration with molecular shieve $3 \mathrm{~A}$, and further by fractional distillation under vacuum. The purity determined by calorimetric method was $99.86 \%$. Differential thermal analysis (DTA) ${ }^{4)}$ was carried out for this specimen, and the results are reproduced in Fig. 1. Run 1 shows the heating curve of the rapidly quenched sample from liquid

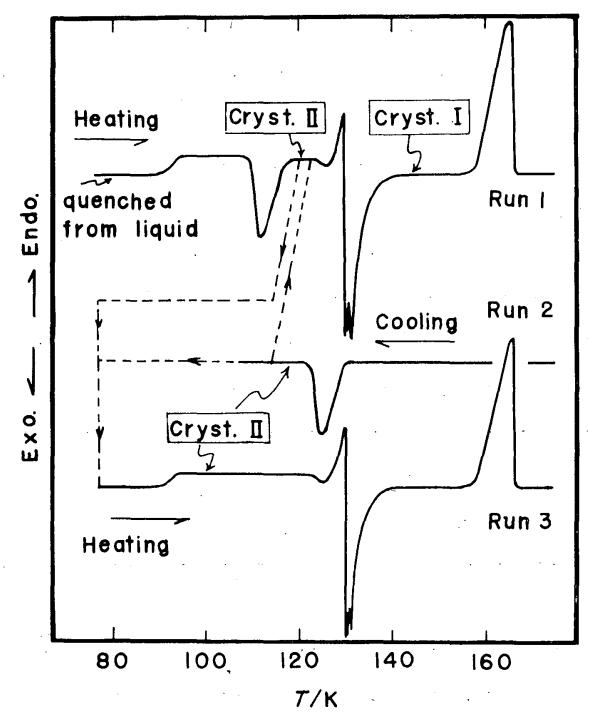

Fig. 1. DTA curves of ethanol. 
by inserting the DTA sample container into liquid nitrogen. The endothermic baseline shift around $95 \mathrm{~K}$ is due to glass transition. Through rather complex thermal behaviors between 100 140 K, the sample was transformed finally into a stable crystalline phase (crystal-I) and melted at $159 \mathrm{~K}$. Run 2 shows the cooling curve from liquid with the cooling rate of $\sim-2 \mathrm{~K} \mathrm{~min}^{-1}$. The supercooled liquid was found to crystallize into a newly found crystalline phase (crystal-II) around $125 \mathrm{~K}$. Run 3 shows the heating curve of this crystal-II from $78 \mathrm{~K}$. Crystal-II has glass transition phenomenon at about $95 \mathrm{~K}$. The phase produced just above the exothermic anomaly around $110 \mathrm{~K}$ in Run I was identified as the crystal-II by investigating its thermal behavior on heating (see the upward arrow from Run 2 and the downward arrow from Run 1 to Run 3). These results can be summerized as follows: (1) an extremely rapid chilling $(-50 \sim$ $-100 \mathrm{~K} \mathrm{~min}^{-1}$ ) of liquid ethanol yields the glassy state of liquid (which is called "glassy liquid" in this paper). (2) the supercooled liquid transforms into crystal-II if the glassy liquid is heated from below its glass transition temperature, and the crystal-II (which can be produced also by a slow cooling of the liquid) shows another glass transition phenomenon. (3) crystal-II melts at $127 \mathrm{~K}$, but during the melting process the irreversible transition into crystal-I occurs inevitably.

Because it seems to be interesting to compare the thermodynamic properties of the glassy liquid and the glassy crystal of one and the same substance, precise heat capacity measurement by the adiabatic calorimeter ${ }^{7)}$ was performed from 14 to $300 \mathrm{~K}$. The sample of the glassy liquid was prepared in situ by rapid quenching of liquid with direct pouring of the liquid nitrogen into the calorimeter can. The exact cooling rate was not determined but its mean value was $-50 \mathrm{~K}$ $\min ^{-1}$ at around $100 \mathrm{~K}$. By this procedure we could obtain the pure glassy liquid. This was proved by the concordance of the measured heat capacities as well as enthalpy just above $\mathrm{Tg}$ with the extrapolated values from the data of the supercooled liquid near the melting point of crystal-I. To prepare the glassy crystal-II, it was necessary to control the cooling rate, since too slow cooling rate gave rise to the crystallization into crystal-I at around $145 \mathrm{~K}$. Since the glassy crystal-II was prepared under the observations of the temperature change as well as the plot of the time derivative of the temperatures, we could ascertain that the supercooled liquid was transformed into crystal-II.

The results of the heat capacity measurement are shown in Fig. 2. The previous results for glassy ethanol by Parks ${ }^{1)}$ and Kelley ${ }^{2)}$ coincide well with that for the glassy crystal in this work. The glass 


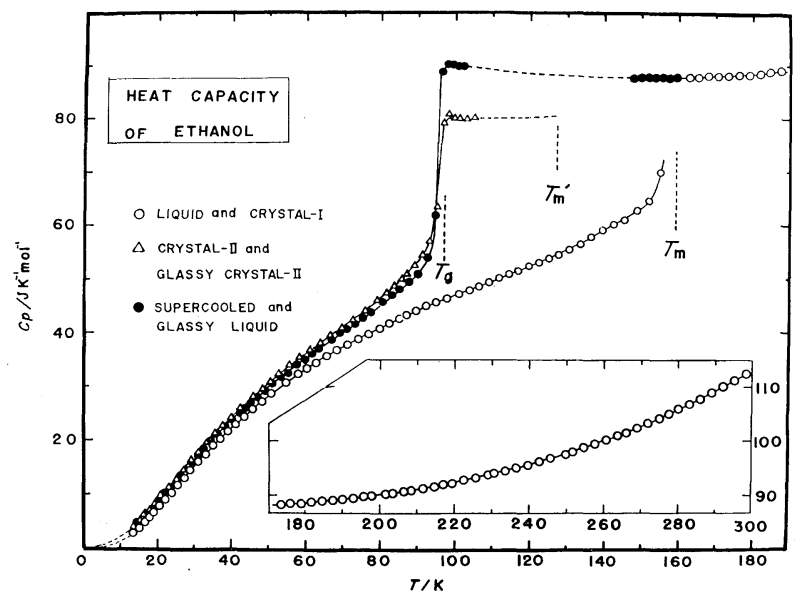

Fig. 2. Heat capacity vs. temperature curves of ethanol.

transition temperature $(T g)$, heat capacity jump at $T g$, and the residual entropy at $0 \mathrm{~K}$ were found to be $97 \mathrm{~K}, 12.85 \mathrm{JK}^{-1} \mathrm{~mol}^{-1}$ and $8.93 \mathrm{JK}^{-1} \mathrm{~mol}^{-1}$ for glassy liquid, and $97 \mathrm{~K}, 9.5 \mathrm{JK}^{-1} \mathrm{~mol}^{-1}$ and 4.24 $\mathrm{JK}^{-1} \mathrm{~mol}^{-1}$ for glassy crystal, respectively. We have already reported ${ }^{5)}$ that two kinds of glassy crystal of cyclohexene have almost the same glass transition temperature $(81 \mathrm{~K}$ and $83 \mathrm{~K})$. On the other hand we have also found ${ }^{6)}$ that the glass transition temperature of glassy liquid of cyclohexene was only $14 \mathrm{~K}$ lower than that of glassy crystal. In this respect it is not so peculiar that the glass transition temperatures of glassy liquid and glassy crystal of ethanol are almost identical. In passing it may be noted here that crystal-II is regarded as the plastic crystal ${ }^{8)}$ with reference to the small entropy of fusion (5.19 $\left.\mathrm{JK}^{-1} \mathrm{~mol}^{-1}\right)$, and also that this crystal-II is always metastable phase under one atmospheric pressure.

From these results it is concluded that both the glassy liquid and the glassy crystal states were studied quantitatively for one and the same substance for the first time. All the previous measurements ${ }^{1,2)}$ for glassy ethanol are not corresponding to the glassy liquid but to the glassy crystal. Finally, it may be pointed out that careful and well defined thermal treatment is indispensable to the study of glassy states in general. The authors wish to express their sincere thanks to Dr. Keiichiro Adachi for his useful discussions about the results of DTA.

\section{References}

1) G. E. Gibson, G. S. Parks, and W. M. Latimer: J. Amer. Chem. Soc., 42, 1542 (1920); G. S. Parks: J. Amer. Chem. Soc., 47, 338 (1925). 
2) K. K. Kelley: J. Amer. Chem. Soc., 51, 779 (1929).

3) K. Adachi, H. Suga, and S. Seki: Bull. Chem. Soc. Japan, 41, 1073 (1968); 43, 1916 (1970) ; 44, 77 (1971) ; 45, 1960 (1972).

4) H. Suga, H. Chihara, and S. Seki: Nippon Kagaku Zasshi, 82, 24 (1961).

5) O. Haida, H. Suga, and S. Seki: Preprint of the 7th Japanese Calorimetry Conference, p. 48 (1971).

6) to be published.

7) H. Suga and S. Seki: Bull. Chem. Soc. Japan, 38, 1000 (1965).

8) J. Timmermans: J. Phys. Chem. Solids, 18, 1 (1961). 